\title{
A Large Vaginal Bulge Might Not Be a Genital Prolapse
}

\section{Natasha Ferreira Teixeira Meletti, Susana Cristina Aidé Viviani Fialho, Fabiana Resende Rodrigues, Carlos Augusto Faria}

Hospital Universitário Antônio Pedro, Universidade Federal Fluminense (UFF), Rio de Janeiro (RJ), Brazil

Email: natashameletti@yahoo.com.br, suaide@oi.com.br,drafabianaresende@gmail.com,

carlosfaria@vm.uff.br/carlosfaria1965@gmail.com

How to cite this paper: Meletti, N.F.T., Fialho, S.C.A.V., Rodrigues, F.R. and Faria, C.A. (2018) A Large Vaginal Bulge Might Not Be a Genital Prolapse. Open Journal of Obstetrics and Gynecology, 8, 362-367. https://doi.org/10.4236/ojog.2018.84040

Received: March 4, 2018

Accepted: April 13, 2018

Published: April 16, 2018

Copyright $\odot 2018$ by authors and Scientific Research Publishing Inc. This work is licensed under the Creative Commons Attribution International License (CC BY 4.0).

http://creativecommons.org/licenses/by/4.0/ (c) (i) Open Access

\begin{abstract}
Vaginal tumors, whether benign or malignant, are rare. They include fibroepithelial polyps (FEPs), which are benign lesions originating in mesenchymal cells, comprised of a core of connective tissue covered by squamous epithelium. They are usually small and asymptomatic. When symptomatic or very large, they may cause bleeding, genital discomfort or the presence of a bulge in the vagina. In the last case, they may be mistaken for a genital prolapse. Although their physiopathology is still not clearly understood, the presence of hormonal receptors and the occurrence of FEPs during the use of hormone therapy or pregnancy suggest that changes in the stroma of these lesions may be induced by hormones. We report on the case of a patient who presented with a vaginal bulge and was referred to the urogynecology outpatient ward with a diagnosis of genital prolapse, which had actually a large fibroepithelial polyp on the posterior vaginal wall.
\end{abstract}

\section{Keywords}

Diagnosis, Pathology, Pelvic Organ Prolapse, Vaginal Neoplasms

\section{Introduction}

Complaints of vaginal bulging are associated with genital prolapse diagnosis. Large vaginal bulging is associated with advanced stage of prolapse, reaching beyond the hymenal ring (POP-Q stages II-IV), but other conditions could be considered in the differential diagnosis [1].

Fibroepithelial polyps (FEPs) are benign tumors originating in mesenchymal cells, comprised of a core of connective tissue covered by squamous epithelium [2]. In the rare cases of FEP occurring in the vagina, they are usually small and 
their diagnosis is due to happenstance during gynecologic examination. When symptomatic or very large, they may cause bleeding, genital discomfort or symptoms comparable to those of a genital prolapse, i.e., the presence of a vaginal bulge [3] [4].

FEPs may start on any of the vaginal walls, including the vaginal vault in women who have had a hysterectomy [2] [4]. It has been reported that FEPs may develop as the result of granulated tissue reaction following some type of lesion in the vaginal mucosa [2] [4] [5]. A delay in the differentiation of fibroblastic cells may explain the emergence of granulated tissue which, occasionally, does not contract properly and becomes a polyp. Besides, some studies support the hypothesis that female steroidal hormones are related to the development of fibroepithelial polyps, and hormonal factors may modulate their growth [2] [4].

Since vaginal tumors are rare, reports in the literature generally involve cases or series of cases and emphasize the differential diagnosis with malignant mesenchymal tumors on the vaginal wall [5] [6].

The objective of the present report is to emphasize that vaginal tumors, although uncommon, should be considered in the differential diagnosis of vaginal bulges.

\section{Case Description}

A 52-year-old woman, gesta 5 para 3 (vaginal deliveries), referring menopause at the age of 30, presented at the urogynecology outpatient ward at the Antônio Pedro University Hospital with a presumed diagnosis of genital prolapse. She complained of a painless, vaginal bulge for approximately three years, which had progressively grown until it was exposed in the vaginal introitus.

In her first visit, it was observed a bulge of approximately $5 \mathrm{~cm}$ in diameter, covered by vaginal epithelium, suggestive of prolapse. Examination with the speculum revealed that the bulge was, actually, a round, tumorous, sessile mass, with a uniform surface, covered by vaginal epithelium, which had its origin in the distal third of the posterior vaginal wall (Figure 1). There was no prolapse. The tumorous mass was fibroelastic and painless when moved. Rectal examination did not show abnormalities or compression due to the mass.

As the patient demanded surgical treatment and had a history of hypertension, angina pectoris and severe coronaropathy she was referred to preoperatory evaluation and laboratory examinations were requested. For this reason, and because of poor socioeconomic status, she returned for a visit only three months later. Upon physical examination, we found the formation of a pedicle, and the lesion hanging down from the lower third of the posterior vaginal wall.

Due to the presence of the comorbidities cited above, resection of the lesion was recommended under local anesthesia with sedation. The procedure was performed without complications. The tissue was sent for histopathological and immunohistochemical analysis.

Under macroscopy, the biopsy showed whitish, smooth, elastic polypoid tissue, 


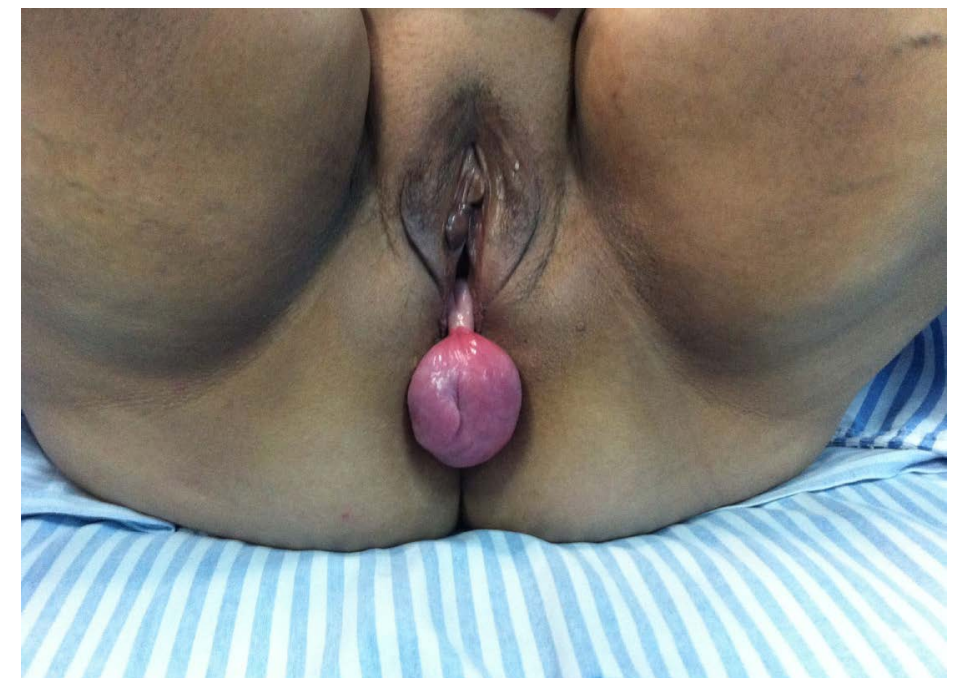

Figure 1. Fibroepithelial polyp arising from the posterior vaginal wall.

weighing $50 \mathrm{~g}$ and measuring $5.0 \times 5.0 \times 4.0 \mathrm{~cm}$. The pedicle measured $1.0 \times 0.8$ $\times 0.4 \mathrm{~cm}$. Cuts on the surface showed homogeneous, light brown, vitreous and elastic tissue. Examination under the microscope showed that the polyp was covered with stratified, squamous epithelium, and the stroma was typically fibroelastic, edematous myxoid and hypocellular, presenting bland stellate cells and occasionally multinucleated stromal giant cells (Figure 2). It had also a central fibrovascular core, containing large and small blood vessels but no malignancy.

The tissue was also subjected to an immunohistochemical analysis, which showed immunoreactivity with immunoexpression of estrogen receptors (ER), progesterone receptors (PR), vimentin, desmin and smooth-muscle actin (SMA), and negative immunoexpression with h-Caldesmon. We concluded that this was a case of a fibroepithelial (stromal) polyp.

The patient has been followed at the outpatient ward since then and has not shown relapse. She has been informed about the importance of reporting on the case and signed an Informed Consent Form.

\section{Discussion}

Although FEPs are normally small, they may become large and result in symptoms of vaginal bulging. In tumors that are sessile or found higher on the vaginal wall, only a very meticulous examination will allow the health professional to differentiate a prolapse from a vaginal wall tumor.

When the patient in the case at hand presented for her first visit, she didn't have other complaints than the bulging, the lesion was sessile and located in the lower third of the vaginal posterior wall. For this reason, it could be exposed, suggesting it was a uterine prolapse or a rectocele.

Our findings are quite different from those described in the study of Halvorsen et al., who found that, in most cases, FEP didn't reach more than $3.5 \mathrm{~cm}$, were 


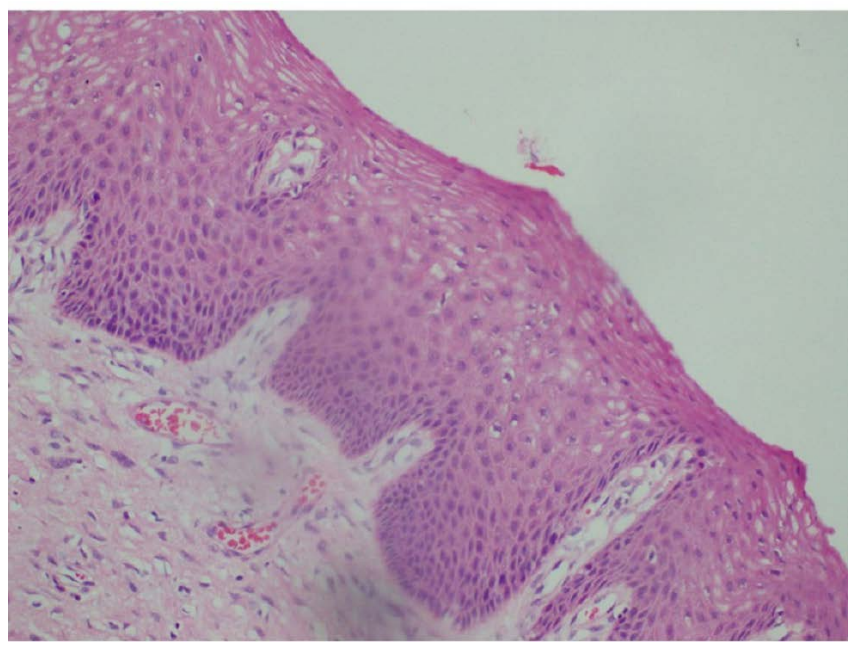

(a)

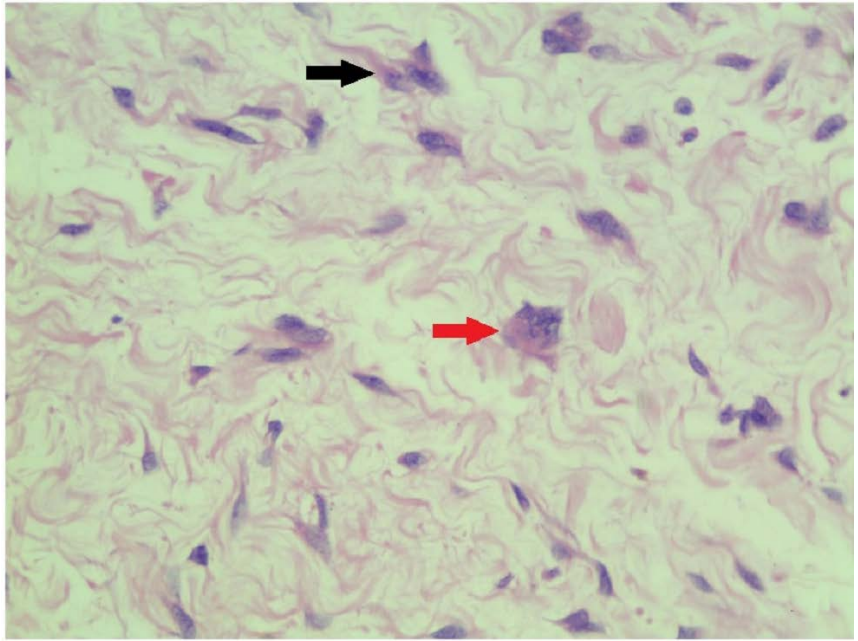

(b)

Figure 2. Fibroepithelial polyp. HE 40×. (a) Epithelial surface exhibiting stratified, squamous epithelium, with fibroelastic, edematous myxoid and hypocellular stroma. HE 20×; (b) Myxoid stroma with bland stellate cells (black arrow) and multinucleated stromal giant cells (red arrow).

discovered incidentally during vaginal examination and had their origin in vaginal vault [2].

The studies of Halvorsen et al., Sharma et al. and Song et al. report that FEPs may develop as the result of granulated tissue reaction following some type of lesion in the vaginal mucosa [2] [4] [5]. In the case at hand, the patient had a history of normal deliveries and an episiotomy scar, which could be the origin of her FEP.

Histologically, the FEP was similar to those included in the study of Halvorsen et al. that presented in most cases multinucleated cells and hypocellular stroma. Besides, the findings of a central fibrovascular core and an overlying squamous epithelium are in accordance with the characteristics described by Nucci for FEP [7]. 
Regarding the immunohistochemical panel, Nucci stated that most of FEP exhibit positivity for vimentin, desmin, estrogen and progesterone receptors [7]. In our case, it was found immunoreactivity for all of them, corroborating the diagnosis of FEP. Although smooth-muscle actin (SMA) is much less frequent, it was described in this patient, but not the biomarker h-Caldesmon, that seems to be more specific for smooth-muscle cells [7].

Some studies suggest that female steroidal hormones are related to the development of fibroepithelial polyps [4]. Our patient, however, had not menstruated for more than 20 years and was not using any type of hormonal therapy. Even though FEPs frequently show expression for estrogen and progesterone receptors, there is insufficient data in the literature to confirm a cause and effect relationship. Besides, all the tissues originating from the urogenital tract have such receptors, and it could explain their finding in this case [8] [9].

In addition to prolapses, other mesenchymal tumors on the vaginal wall, such as botryoid sarcoma, must be considered in the differential diagnosis. This involves a rapid-growth, malignant lesion most frequently located on the lateral vaginal wall, usually similar in appearance to a bunch of grapes or with various finger-like formations [6].

Since FEP is a benign tumor, mere excision is usually curative. Relapse is extremely rare and mainly occurs when excision is not complete [10].

\section{Conclusion}

In conclusion, we can say that, although complaints of genital bulging are often related to genital prolapse, they may also be caused by tumors on the vaginal walls, as FEPs. For greater diagnostic accuracy, it is important to bear this possibility in mind and perform the examination meticulously. Nonetheless, a final diagnosis of FEP will depend on resection of the lesion, with histopathological and immunohistochemical analyses.

\section{Acknowledgements}

We appreciate the patient who consented to the description about this case.

\section{References}

[1] Haylen, B.T., de Ridder, D., Freeman, R.M., Swift, S.E., Berghmans, B., Lee, J., et al. (2010) An International Urogynecological Association (IUGA)/International Continence Society (ICS) Joint Report on the Terminology for Female Pelvic Floor Dysfunction. International Urogynecology Journal, 21, 5-26.

https://doi.org/10.1007/s00192-009-0976-9

[2] Halvorsen, T.B. and Johannesen, E. (1992) Fibroepithelial Polyps of the Vagina: Are They Old Granulation Tissue Polyps? Journal of Clinical Pathology, 45, 235-240. https://doi.org/10.1136/jcp.45.3.235

[3] Rollason, T.P., Byrne, P. and Williams, A. (1990) Immunohistochemical and Electron Microscopic Findings in Benign Fibroepithelial Vaginal Polyps. Journal of Clinical Pathology, 43, 224-229. https://doi.org/10.1136/jcp.43.3.224

[4] Sharma, S., Albertazzi, P. and Richmond, I. (2006) Vaginal Polyps and Hormones- 
Is There a Link? A Case Series. Maturitas, 53, 351-355. https://doi.org/10.1016/j.maturitas.2005.06.007

[5] Song, J.S., Song, D.E., Kim, K.-E. and Ro, J.Y. (2012) Cellular Pseudosarcomatous Fibroepithelial Stromal Polyp of the Vagina during Pregnancy: A Lesion That Is Overdiagnosed as a Malignant Tumor. Korean Journal of Pathology, 46, 494-498. https://doi.org/10.4132/KoreanJPathol.2012.46.5.494

[6] O’Quinn, A.G., Edwards, C.L. and Gallager, H.S. (1982) Pseudosarcoma Botryoides of the Vagina in Pregnancy. Gynecologic Oncology, 13, 237-241. https://doi.org/10.1016/0090-8258(82)90032-4

[7] Nucci, M.R. (2003) Application of Biomarkers in the Diagnosis and Differential Diagnosis of Uterine and Vulvar Mesenchymal Tumours. Current Diagnostic Pathology, 9, 26-38. https://doi.org/10.1054/cdip.2003.0153

[8] Iosif, C.S., Batra, S., Ek, A. and Astedt, B. (1981) Estrogen Receptors in the Human Female Lower Urinary Tract. American Journal of Obstetrics \& Gynecology, 141, 817-820. https://doi.org/10.1016/0002-9378(81)90710-9

[9] Batra, S.C. and Iosif, C.S. (1987) Progesterone Receptors in the Female Lower Urinary Tract. The Journal of Urology, 138, 1301-1304. https://doi.org/10.1016/S0022-5347(17)43588-9

[10] Orosz, Z., Lehoczky, O., Szoke, J. and Pulay, T. (2005) Recurrent Giant Fibroepithelial Stromal Polyp of the Vulva Associated with Congenital Lymphedema. $G y$ necologic Oncology, 98, 168-171. https://doi.org/10.1016/j.ygyno.2005.01.020 